\title{
FIBROUS DYSPLASIA OF MANDIBLE: A CASE REPORT
}

\author{
Saurabh Vishwanath Saxena ${ }^{1}$, Hardik Kishor Ganatra²
}

${ }^{1}$ Resident, Department of Plastic Surgery, VS General Hospital, NHL Medical College, Ahmedabad. ${ }^{2}$ Resident, Department of Plastic Surgery, VS General Hospital, NHL Medical College, Ahmedabad.

\begin{abstract}
Craniofacial fibrous dysplasia is one of the three types of fibrous dysplasia that can affect the bones of the craniofacial complex, including the mandible and maxilla. Fibrous dysplasia is a non-neoplastic hamartomatous developmental fibro-osseous lesion of the bone-forming mesenchyme that manifests as a defect in osteoblastic differentiation and maturation. It is a lesion of unknown aetiology, uncertain pathogenesis, and diverse histopathology. Fibrous dysplasia represents about $5 \%$ of all bone tumours and over $7 \%$ of all benign tumours of body. Monostotic fibrous dysplasia of the mandible is an unusual manifestation of the disease that is usually benign, occurs in young individuals, and is managed by conservative curettage or debridement. We present a rare case of unilateral fibrous dysplasia of left side of mandible. The clinical diagnostic approach including imaging studies: Orthopantomogram $(\mathrm{OPG})$ and 3D tomography is described. Histological examination served an adjunctive role; however, the final diagnosis was obtained after excision of the swelling and confirming it by histopathological examination and it was diagnosed as benign fibrous dysplasia.
\end{abstract}

\section{KEYWORDS}

Fibrous Dysplasia, Hamartoma, Monostotic, Polyostotic, Ameloblastoma, Cementoblastoma, Osteoblastoma.

HOW TO CITE THIS ARTICLE: Saxena SV, Ganatra HK. Fibrous dysplasia of mandible: a case report. J. Evolution Med. Dent. Sci. 2016;5(94):6972-6975, DOI: 10.14260/jemds/2016/1577

\section{INTRODUCTION}

Fibrous dysplasia (FD) is a bone development anomaly characterised by extensive hamartomatous proliferation of fibrous tissue within the medullary bone, with secondary bony metaplasia, producing immature, newly formed and weakly calcified bone, without maturation of the osteoblast which appears radiolucent on radiographs, with the classically described ground-glass appearance, leading to osteolytic lesion, fractures and deformations.(1)

It is a benign bone disorder of an unknown aetiology, uncertain pathogenesis and diverse histopathology. Fibrous dysplasia represents about $5 \%$ of all bone tumours and over $7 \%$ of all benign tumours of body.

Cranial or facial bones are affected approximately in 30\% of the patients. The average age of the patients with FD is 25 years (from 5 to 67 ) without sex preference and usually manifests before the 3rd decade of life.(2)

Fibrous dysplasia is described in terms of three major types: monostotic, involving a single bone; polyostotic, having multiple lesions involving multiple bones; and McCune Albright syndrome, a polyostotic form of fibrous dysplasia that also involves endocrine abnormalities.

The monostotic form of fibrous dysplasia is the most common, comprising $70 \%$ of cases, most likely quiescent at puberty. Deformity is progressive and by mass effect there may be impingement on other structures and functional impairment. The clinical findings are asymptomatic, facial asymmetry, loss teeth and facial deformity.

Financial or Other, Competing Interest: None.

Submission 12-03-2016, Peer Review 22-04-2016,

Acceptance 28-04-2016, Published 24-11-2016.

Corresponding Author:

Dr. Saurabh Vishwanath Saxena,

FF- 4, Datt Flats, Beside Hindu Panjabi Hall,

Near Home Science College,

Napier Town, Jabalpur-482001,

Madhya Pradesh.

E-mail: saurabh197832@yahoo.in

DOI: $10.14260 / \mathrm{jemds} / 2016 / 1577$
The complications of the lesions involving sphenoid, orbital, frontal bones, are proptosis, visual disturbances, facial asymmetry and orbital dystopia. The fifth nerve impairment, hearing loss and seizure disorders have been reported as neurological complications.

Computed tomography and magnetic resonance imaging can be used for differential diagnosis with malignancies.

The generally younger age of onset and its unilateral distribution allows FD to be readily differentiated from Paget's disease which affects older patients and is frequently bilateral. Malignant degeneration occurs in less than $1 \%$ of cases of fibrous dysplasia. Malignancies are almost exclusively osteosarcoma.(3)

In our case, Monostotic Fibrous Dysplasia localised in the left side of mandible that caused bone expansion and patient presented with facial asymmetry.

\section{CASE REPORT}

A 9-year-old male presented at our institute with complaints of pain in the lower jaw without precise localisation and swelling on the left side of mandible. In the history of the patient, the swelling was noticed by his mother one year ago but the patient didn't know when the swelling had occurred. The swelling gradually increased in one year to the present size.

- There was no history of trauma.

- There was no family history with similar findings.

- The general physical examination revealed a moderately built patient with satisfactory vital signs.

On extra-oral examination, a swelling of $4 \mathrm{~cm} \mathrm{x} 4 \mathrm{~cm}$ was present on left side of face extending superior-inferiorly $2 \mathrm{~cm}$ below the cantho-tragal line. Oral examination revealed the presence of unilateral expansion in the distal part of the alveolar ridge of lower jaws .The covering and surrounding mucosa was normal in colour, without any clinical manifestation of inflammation or ulceration.

On palpation, affected areas were nontender, with hard consistency and flat surface and no local rise of temperature. 
Blood \& biochemical investigations showed alkaline phosphatase 87 IU/L, Serum Calcium 9.8 mg\% \& Serum Phosphorus $3.1 \mathrm{mg} \%$ which were within normal range.

The initial diagnosis based on the clinical examination was a bony tumour arising from the left side of jaw.

Imaging Studies with OPG and 3D tomography was undertaken for accurate definition of the bone density and for obtaining the definite diagnosis.

They confirmed lesion confined to the interior of bone with no soft-tissue involvement. Mandibular involvement had a mixed radiolucent and radiopaque pattern. A diagnosis of ameloblastoma was kept by the radiologist after the scan.

Orthopantomogram revealed diffusive radiolucent areas in the posterior part of left mandible. In the area of the lower left second molar above the mandibular canal, radio opacity measuring around $10 \mathrm{~mm}$ was observed. A bone and soft tissue was taken for histological examination. There was no root resorption or displacement of teeth on the affected side.

The histological findings showed fibrous modification of the bone tissue without soft tissue involvement. Multiple diagnoses brought out by the histopathology report were fibro-osseous benign lesion, cementoblastoma, osteoblastoma.

The patient was operated and the swelling was excised along the buccal surface of mandible without disrupting the continuity of lingual side of mandible.

Histopathology report (slide no.- 39339/14/INR no. 27910) has confirmed that this is a benign fibrous dysplasia.

The post-operative course of the patient was uneventful.

The patient was discharged and advised to follow up regularly for examination and to observe and inform for any change in growth formation and appearance of pain.

The level of alkaline phosphatase was serially measured on follow-up.

Three monthly follow-up revealed a healthy scar line without recurrence of the swelling, and with normal alkaline phosphatase levels.

\begin{tabular}{|c|c|}
\hline Time Interval & $\begin{array}{c}\text { Alkaline Phosphatase } \\
\text { Levels }\end{array}$ \\
\hline Pre-operative & $92 \mathrm{u} / \mathrm{L}$ \\
\hline $\begin{array}{c}\text { Immediate Post- } \\
\text { operative }\end{array}$ & $67 \mathrm{u} / \mathrm{L}$ \\
\hline Three monthly follow-up & $56 \mathrm{u} / \mathrm{L}$ \\
\hline
\end{tabular}

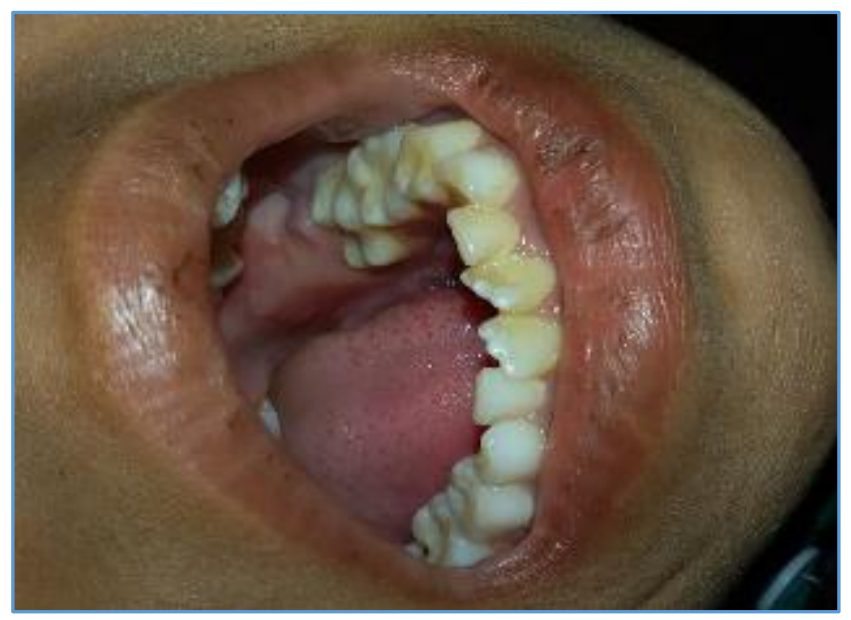

Fig. 1

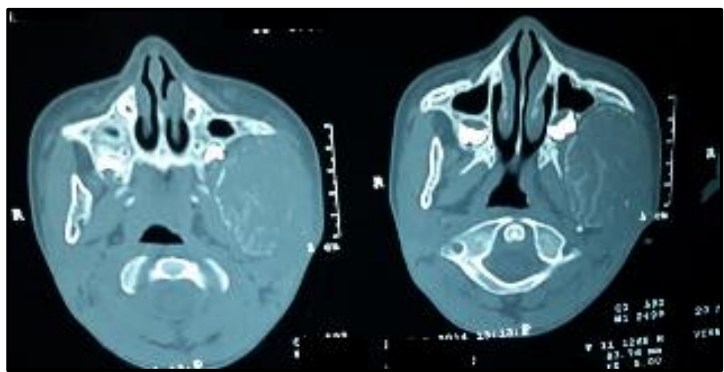

Fig. 2

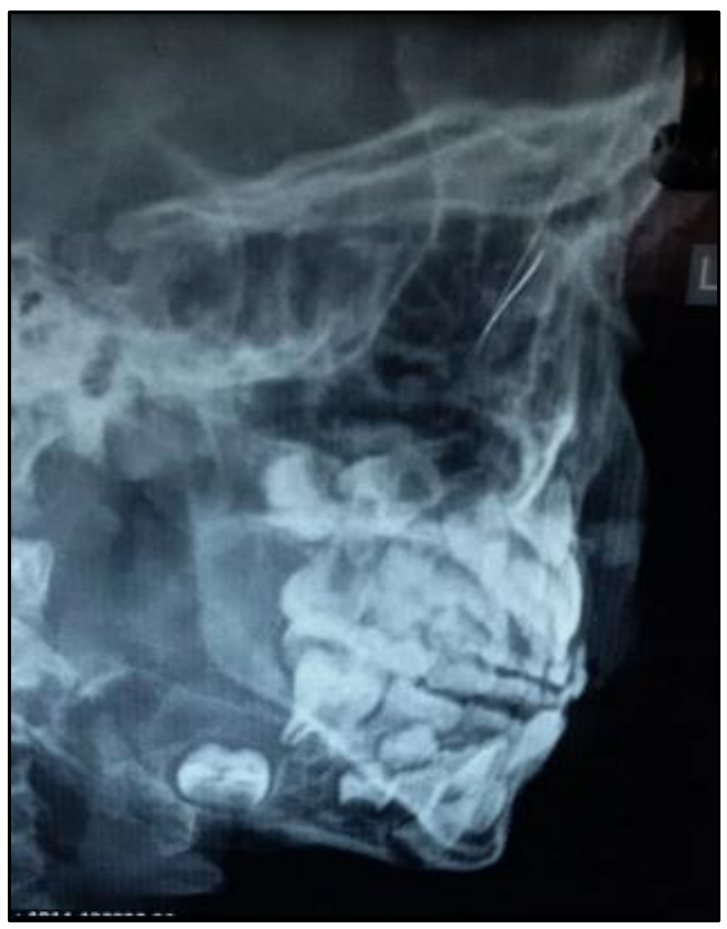

Fig. 3

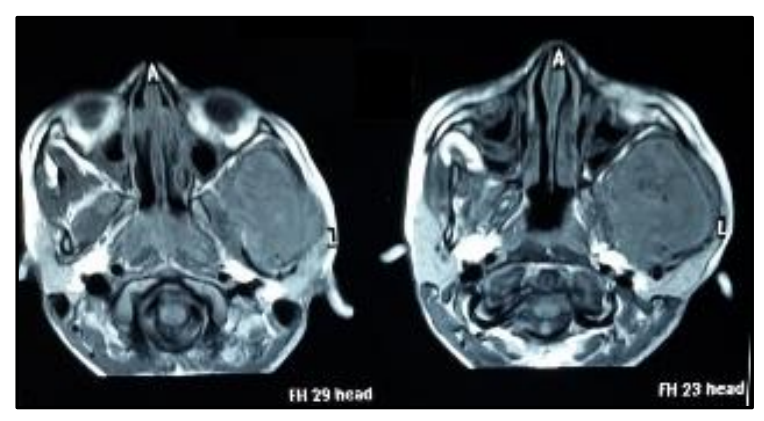

Fig. 4

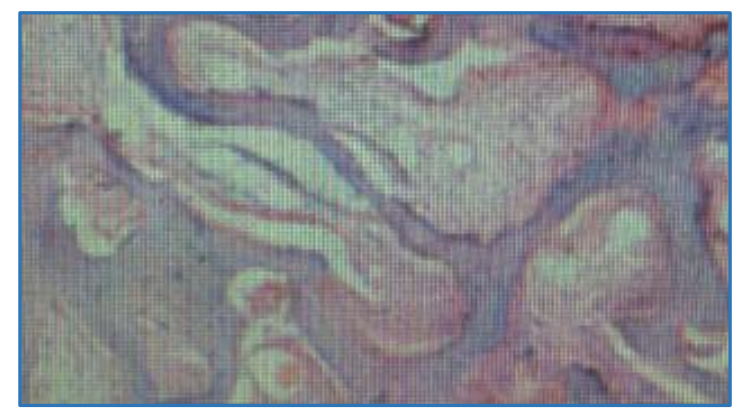

Fig. 5 


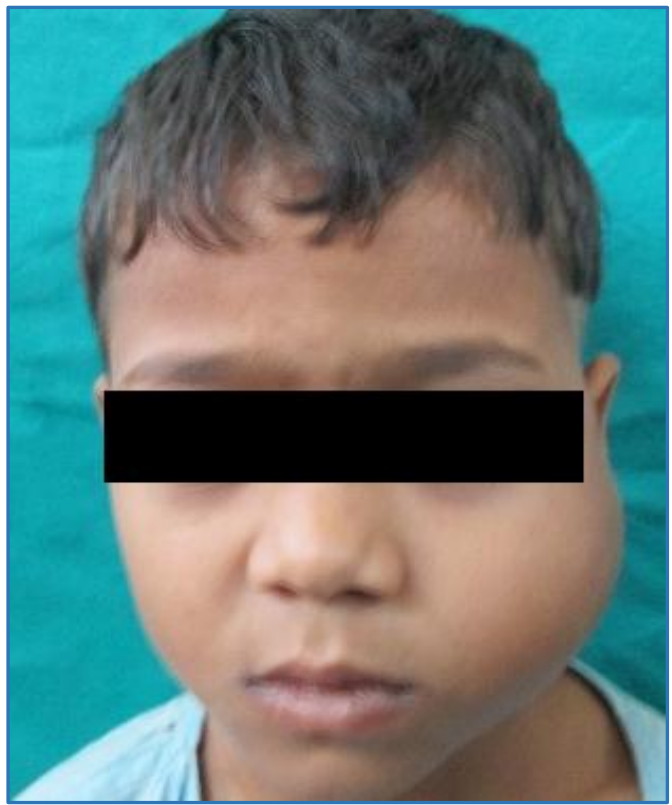

Fig. 6

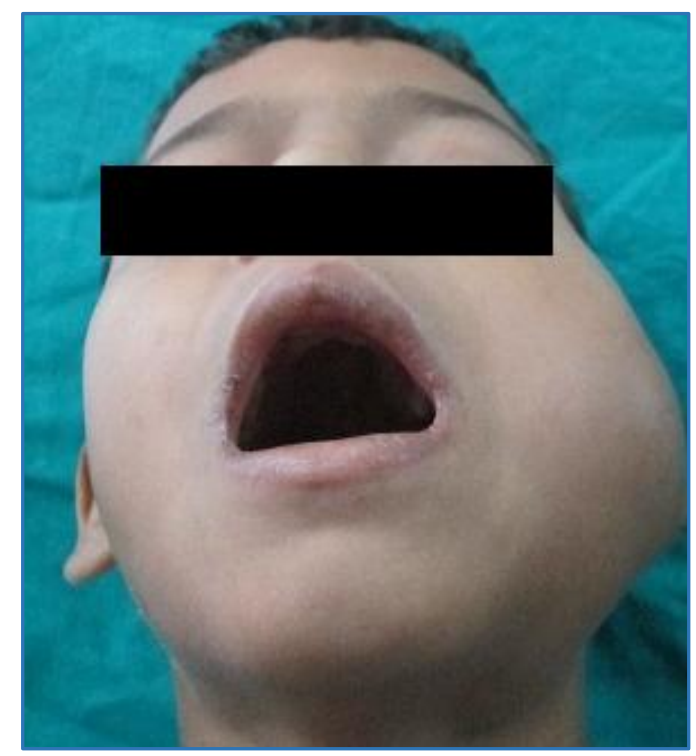

Fig. 7

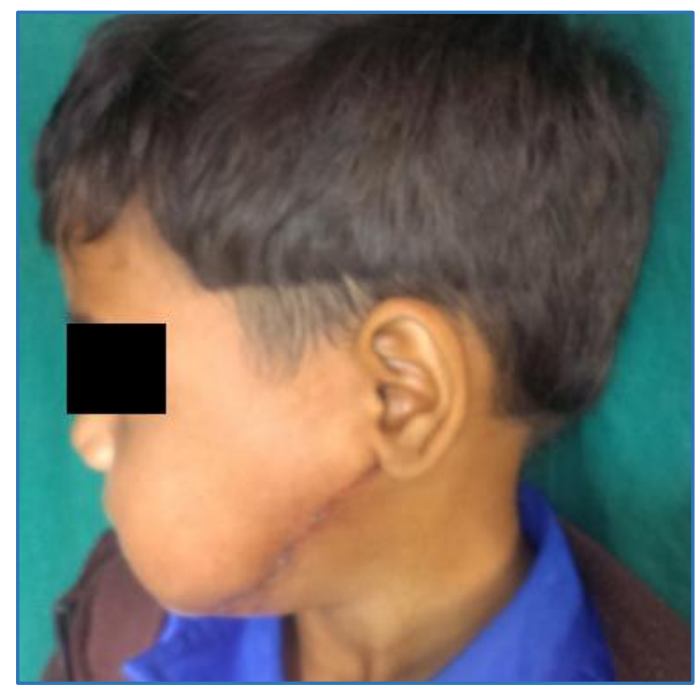

Fig. 8

\section{DISCUSSION}

Fibrous dysplasia of the cranium is a rare disorder of unknown aetiology in which normal bone is replaced by abnormal fibroconnective tissue proliferation. Mandibular involvement may threaten the functions of mastication, speech, respiration and facial and jaw cosmetics. Therefore, the disease progress is of utmost importance.

Ozek et al(4) reported in the series of 16 patients with Fibrous Dysplasia of the craniomaxillofacial bones, one patient was in his first decade, 11 patients were in their second decade, 3 patients were in their third decade and one patient in the series was in his fourth decade when the symptoms occurred.

Keijser et al(5) reported the case with 13 patients after 20 years of age, the two of cases are polyostotic and the rest of cases are monostotic.

In our case, age of the patient was 9 years. The patient at present is in quiescence and no bone increase is detected during previous years. The pain in the lower jaw, being the main complaint of the patient, is considered to be caused by compression of the mandible nerve of the enlarged fibrous tissue.

As no one investigation is pathognomonic, the diagnosis only becomes apparent after collation of history, examination, histology and radiology.

The differential diagnosis with similar radiographic appearance such as ameloblastoma, central giant cell granuloma, odontogenic cyst, ossifying fibroma, osseous dysplasia, chronic sclerosing osteomyelitis and osteosarcoma should be considered.

Surgical excision of the affected bone tissue is usually a successful way of treatment. However, it leads to a huge functional and aesthetic deficit, as well as long-term postoperative complications. $(5,6,7)$

Because radical surgery would possibly increase morbidity by removal of teeth and excision of bone, hence, conservative treatment has been treatment of choice. Shaving and debridement of lesion are parts of conservative treatment. Surgical approach aims: stable occlusion, facial aesthetics and evasion of post-operative relapse. The radiotherapy is contraindicated. Bisphosphonates are used in cases when an intervention is necessary but the surgery cannot be performed.(6,7)Treatment of mandibular defects is complex and includes free vascularised flaps. Donor areas involve the iliac crest, radius, scapula and fibula. Taylor et al(8)firstly reported the transfer of the free fibula flap.

In 1989, the method was used for the segmental mandibulectomy defects by Hidalgo.(9) The fibular flap is considered as one of the ideal flaps for long mandibular defects and is superior to the iliac crest for this purpose, especially in older patients.

Munoz Guerra et al reported 26 cases with using vascularised free fibular flap for mandibular reconstruction. Because patients with FD may be at risk of malignant transformation, periodic follow-up is mandatory to detect such transformation.(10)Recurrence of FD is rare when the lesion has occurred in adults but it is seen more commonly in growth period.

In our patient, excision of the entire swelling was done. The inner table of mandible was kept intact and primary closure 
was done. The wound healed well with an acceptable scar. No recurrence so far has been noted.

\section{CONCLUSION}

Isolated cases of fibrous dysplasia in mandibular region are rare and can be difficult to differentiate from other benign and malignant bone disorders. Such cases usually present to dental surgeons and referred to plastic surgeon for further management. Hence, sufficient knowledge on this condition is important for the proper diagnosis, treatment and prevention of further complications. Surgical excision of the tumour is usually a successful way of treatment.

Our case with its clinical and radiographic features represents an addition to the literature of monostotic fibrous dysplasia.

\section{REFERENCES}

1. Hamida BF, Jlaiel R, Rayana BN, et al. Craniofacial fibrous dysplasia: a case report. J Fr Ophtalmol 2005;28(8):e6.

2. Saglik Y, Atalar H, Yildiz Y, et al. Management of fibrous dysplasia. A report on 36 cases. Acta Orthop Belg 2007;73(1):96-101.
3. Parekh SG, Donthineni-Rao R, Ricchetti E, et al. Fibrous dysplasia. J Am Acad Orthop Surg 2004;12(5):305-13.

4. Ozek C, Gundogan H, Bilkay U,et al. Craniomaxillofacial fibrous dysplasia. J Craniofac Surg 2002;13(3):382-9.

5. Keijser LC, van Tienen TG, Schreuder HW, et al. Fibrous dysplasia of bone: management and outcome of 20 cases. J Surg Oncol 2001;76(3):157-68.

6. Becelli R, Perugini M, Cerulli G,et al. Surgical treatment of fibrous dysplasia of the craniomaxillofacial area. Review of the literature and personal experience form 1984 to 1999. Minerva Stomatol 2002;51(7-8):293-300.

7. Peled M, El-Naai IA, Lipin Y, et al. The use of free fibular flap for functional mandibular reconstruction. J Oral and Maxillofac Surg 2005;63(2):220-4.

8. Taylor GI, Miller GD, Ham FJ. The free vascularized bone graft. A clinical extension of microvascular techniques. Plast Reconstr Surg 1975;55(5):533-44.

9. Hidalgo DA. Fibula free flap: a new method of mandible reconstruction. Plast Reconstr Surg 1989;84(1):71-9.

10. Valentini V, Cassoni A, Marianetti TM, et al. Craniomaxillofacial fibrous dysplasia: conservative treatment or radical surgery? A retrospective study on 68 patients. Plast Reconstr Surg 2009;123(2):653-60. 